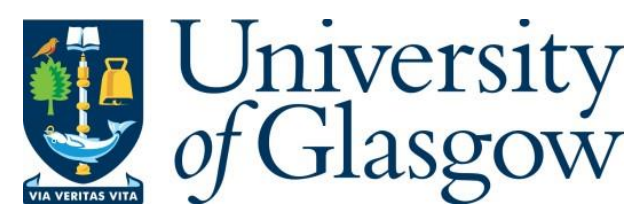

Ozioko, O. O., Hersh, M. and Dahiya, R. (2018) Inductance-based Flexible Pressure Sensor for Assistive Gloves. In: 2018 IEEE Sensors, New Delhi, India, 28-31 Oct 2018, ISBN 9781538647073.

There may be differences between this version and the published version. You are advised to consult the publisher's version if you wish to cite from it.

http://eprints.gla.ac.uk/201381/

Deposited on: 18 November 2019

Enlighten - Research publications by members of the University of Glasgow http://eprints.gla.ac.uk 


\title{
Inductance-based Flexible Pressure Sensor for Assistive Gloves
}

\author{
Oliver Ozioko, Marion Hersh, Ravinder Dahiya* \\ School of Engineering, University of Glasgow, UK \\ *Correspondence to: Ravinder.Dahiya@glasgow.ac.uk
}

\begin{abstract}
This paper presents an inductance-based flexible pressure sensor to support the tactile communication between deafblind people. The pressure sensor was realized with a soft ferromagnetic elastomer and a $17 \mu \mathrm{m}$-thick coil fabricated on a 50 $\mu \mathrm{m}$ thick flexible polyimide sheet. The ferromagnetic elastomer acts as the core of the coil, which when pressed, sees the metal particles moving closer to each other and leads to changes in the inductance. The coil, with $75 \mu \mathrm{m}$ wide wires and $25 \mu \mathrm{m}$ pitch, was realized using LIGA (Lithographie Galvanoformung, Abformung) micro molding technique. Four different sensors have been fabricated using different ratios $(1: 1,2: 1,3: 1$ and 5:1) of ecoflex and iron nanoparticles. The results show that the higher the ratio the better the response of the sensor. The presented sensor is intended to be integrated in a smart glove having dual function of tactile sensing and vibrotactile feedback.
\end{abstract}

Keywords-Pressure sensor; Tactile sensor; Smart Glove; Assistive technology; Inductance; Deafblind Communication

\section{INTRODUCTION}

Our interaction with the real world comes through five basic sensory modalities and mainly through vision, touch and audio [1]. However, this does not work for deafblind people as they have impaired hearing and vision modalities, and hence rely on touch-sensing. This makes tactile interfaces critical for them - to interact with the real world either locally or remotely $[2,3]$. A range of technologies have been explored to assist the deafblind people, including smart communication gloves $[4,5]$. Table I summarises some of the existing assistive communication gloves for deafblind people. Early communication devices for deafblind people include handtapper [6], which uses rigid tactile interface for sending messages and movable pins for vibrotactile feedback. Recent assistive communication gloves include the DB-hand which uses tactile buttons and vibration motors [7]. The buttons are used as tactile interface for composing messages while the vibration motors help to create vibrotactile feedback. Another smart glove (Lorm glove) uses fabric pressure sensors to send messages and vibration motors for tactile feedback [8]. Our previous work uses force sensing resistors as well as commercial vibration motors [9]. Other devices use six capacitive touch sensors and six vibration motors [10] or six switches and six vibration motors [11].

The above smart gloves generally have a set of tactile sensors and actuators, with sensors used to get information about contact feature and the actuators serving as a means of vibrotactile feedback. For improved acceptance of these communication technologies and their effective use the sensors and actuators integrated in these smart gloves should be able to bend or conforms to curvy fingers of deafblind. However, most of the assistive devices available today make use of rigid and/or commercial sensors and actuators which is a challenge when it comes to device flexibility, and conformability. Thankfully, the advances in flexible electronics provide timely opportunities for the development of customisable and conformable devices for use in these assistive gloves [12-15]. Tapping into the opportunities provided by flexible electronics, we present here an inductance-based flexible pressure sensor to be integrated with embedded flexible microactuator in a smart glove. The presented sensor will facilitate the development of a flexible touch-sensitive vibrotactile microactuator with a dual function of sending messages and creating localized vibrotactile feedback. Eventually the assistive communication gloves for deafblind people will be more flexible, customizable and conformable. The proposed microactuator works on electromagnetic principle and hence the motivation for fabricating an inductance-based pressure sensor. This means that the same coil could be used for both sensing and vibrotactile feedback. Rigid inductance-based pressure sensors have previously been developed for biomedical applications $[16,17]$, but their rigidity is a limitation in this regard.

This paper presents the fabrication of an inductance-based flexible pressure sensor using a flexible coil and a soft and conformable ferromagnetic elastomer. The pressure sensor have been realized with a soft ferromagnetic elastomer and a $17 \mu \mathrm{m}$-thick coil fabricated on a $50 \mu \mathrm{m}$ thick flexible polyimide sheet. Four such sensors were fabricated using different ratios $(1: 1,2: 1,3: 1$ and 5:1) of ecoflex and iron nanoparticles and the results show that the higher the ratio the better the response of the sensor. Fig. 1, shows the layers of the fabricated sensor, the layers of the proposed touch-sensitive microactuator and the overall concept of assistive glove. Only the touch-sensitive part of this vibrotactile microactuator is presented here.

The paper is organized as follows: The structure of the fabricated sensor and its operating principle are described in Section II. This is followed by the description of fabrication steps in Section III. The sensor characterization and experimental results are presented in Section IV. Finally, the paper is concluded with a summary of the results and future outlook.

TABLE I. Summary of ASSistive DeafBlind Communication Gloves

\begin{tabular}{|l|l|l|l|l|l|}
\hline \multirow{2}{*}{$\begin{array}{c}\text { Assistive } \\
\text { Glove }\end{array}$} & \multicolumn{2}{|c|}{ Tactile Sensor } & \multicolumn{2}{c|}{ Actuator } & Ref. \\
\cline { 2 - 6 } & \multicolumn{1}{c|}{ Type } & Num. & \multicolumn{1}{c|}{ Type } & Num. & \\
\hline Lorm Glove & Fabric Pressure sensor & None & Vibration motors & $32^{\text {ab }}$ & {$[8]$} \\
\hline DB-Hand & Tactile Button & $>10^{\text {ab }}$ & Vibration motors & $>10^{\text {ab }}$ & {$[7]$} \\
\hline $\begin{array}{l}\text { SmartFingerB } \\
\text { raille }\end{array}$ & Force Sensing Resistor & $6^{\mathrm{b}}$ & Vibration motors & $6^{\text {ab }}$ & {$[9]$} \\
\hline Handtappper & Keyboard interface & None & Movable pins & $14^{\text {ab }}$ & {$[6$,} \\
\hline Comm. Glove & Capacitive & $6^{\mathrm{a}}$ & Vibration motors & $6^{\text {ab }}$ & {$[10]$} \\
\hline Body Braille & Switches & $8^{\text {ab }}$ & Vibration motors & $6^{\text {ab }}$ & {$[1]$} \\
\hline
\end{tabular}




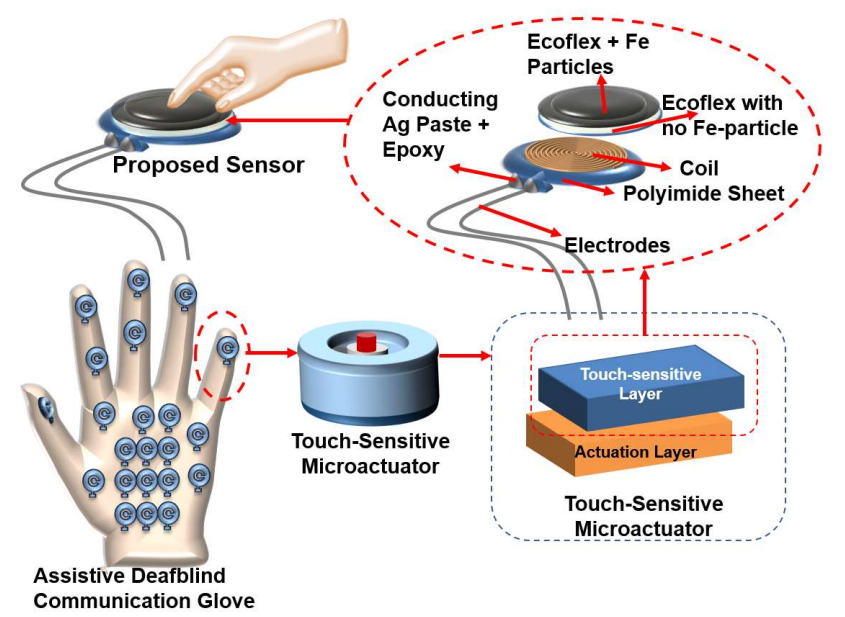

Fig. 1.Sensor structure and the concept of assistive communication glove for deafblind people.

\section{SENSOR STRUCtURE AND Operating PRINCIPLE}

The sensor has two main parts: (1) a spiral coil, and (2) a ferromagnetic elastomer made with Ecoflex and iron particles. The sensor works through a change of inductance which occurs when a force is applied on it. Every coil carrying current has a characteristic inductance and bringing a ferromagnetic material (like soft iron) close to it changes this inductance. In this work, a flexible spiral coil was fabricated on a flexible polyimide substrate and an elastomer mixed with iron particles serves both as separator and ferromagnetic material. This means that one part of the elastomer contains dense iron particles while the other part is nearly without Fe-particles. The part with very few iron particles are placed directly on the coil and serves as the separator between the coil and the dense iron particles. A force on the elastomer reduces the distance between the iron particles and the coil thereby changing the inductance of the coil. The motivation for having a partially iron-filled elastomer with iron particles is to reduce the fabrication steps and the need for an additional separation layer

\section{SENSOR FABRICATION}

The sensor Fabrication involves two main processes: (1) Fabrication of coil, and (2) Fabrication of the Ferromagnetic elastomer. These are described below.

\section{A. Fabrication of Coil}

The coil was fabricated using the LIGA (Lithographie Galvanoformung, Abformung) process, as shown in Fig. 2. The motivation for using the LIGA is to increase the thickness of the coil using standard and low-cost electroplating method. $20 \mathrm{~nm} / 50 \mathrm{~nm} \mathrm{NiCr} / \mathrm{Au}$ seed layer was deposited on a flexible $50 \mu \mathrm{m}$ polyimide sheet using Plassys MEB 550S Electron Beam Evaporator system. An AZ4562 photoresist was spun on the surface of the deposited metal at 2000rpm for 3 seconds. The sample was left at room temperature for 30 minutes to allow the solvent to evaporate before baking so as to avoid trapped bubbles. The sample was then baked with a hotplate at $100^{\circ} \mathrm{C}$ for 10 minutes and again left for 30 minutes before UV

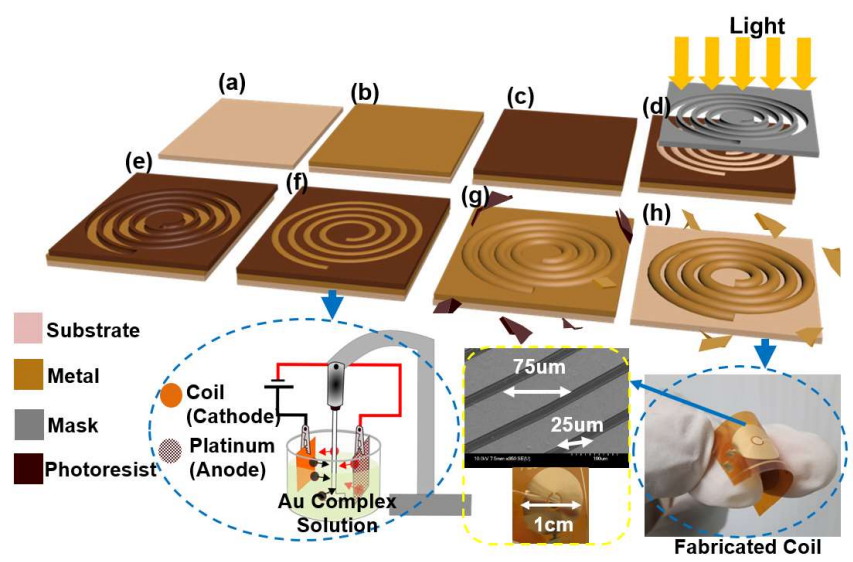

Fig. 2. Fabrication steps for coil: (a) Initial flexible substrate, (b) Deposit gold, (c) Spin coat photoresist, (d) Expose photoresist, (e) Develop the photoresist, (f) Electroplate the coil, (g) lift-off the photoresist, and (h) lift of the seed layer.

(d)

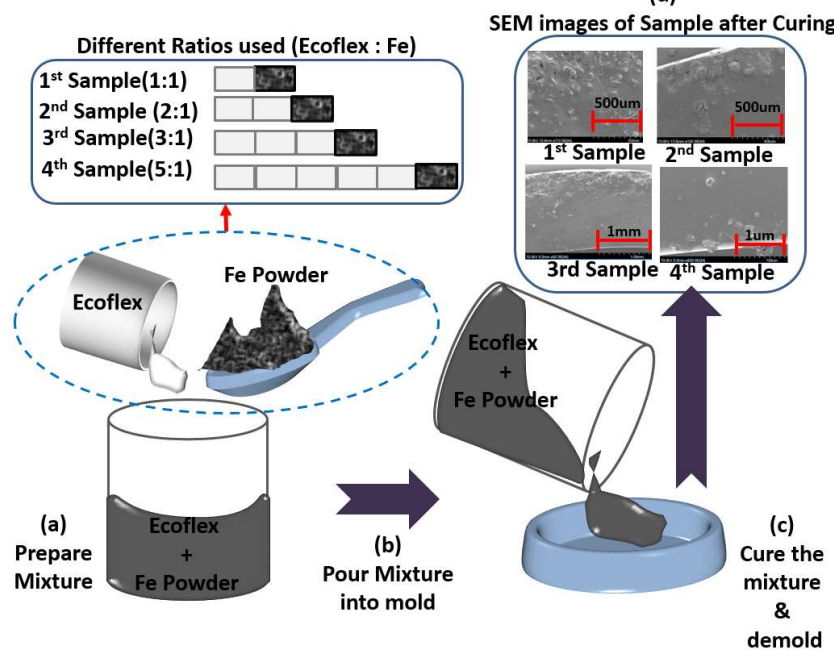

Fig. 3. Fabrication steps for the ferromagnetic elastomer

exposure. The baked sample was then exposed for 60 minutes under a UV light using standard lithography technique. The exposed photoresist was then developed using AZ826 developer for 10 minutes and washed using reverse osmosis water. This is followed by the gold-plating of developed sample for 45 minutes using a non-cyanide gold complex solution connected to the cathode of a constant current source. The seed layer was then etched using Gold $(\mathrm{Au})$ and Nichrome (NiCr) etchant exposing the flexible polyimide sheet with the required coil pattern as shown in Fig. 2(h).

\section{B. Fabrication of Ferromagnetic Elastomer}

The fabrication of ferromagnetic elastomer is shown in Fig. 3. The ferromagnetic elastomer was realized using Ecoflex silicone from Smooth-ON and Iron powder from First4Magnets. Ecoflex was mixed with iron powder in different ratios as shown in Fig. 3. The 1st, 2nd, 3rd and 4th samples were prepared using Ecoflex to Iron ratio of 1:1, 2:1, $3: 1$, and 5:1 respectively. 


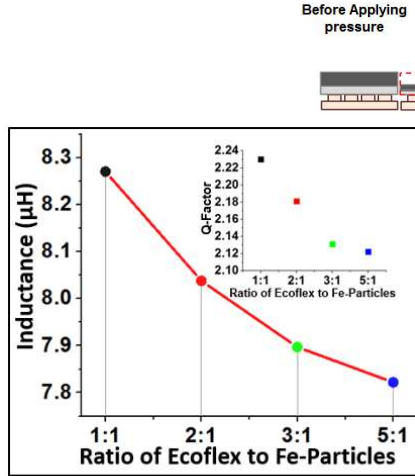

(a)

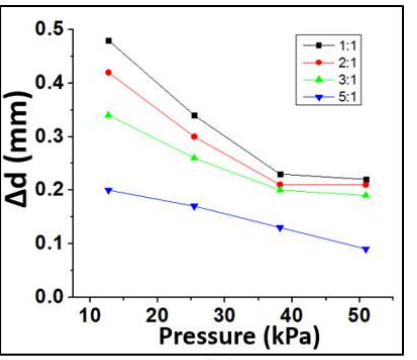

(b)
Fig. 4.(a) Variation of Q-factor and Inductance for the four sensors, (b) Variation of pressure and distance moved by the elastomer.

Each sample was allowed to cure at room temperature for at least 4 hours. During curing, the Fe-nanoparticles settled down giving rise to an elastomer that serves both as the ferromagnetic material as well as the dielectric separator.

\section{SENSOR CHARACTERISATION AND RESUlTS}

The sensor characterization was carried out with a vertical linear stage driven by a linear motor with an attached square contactor which applies the force on the sensor. The position of the motor was controlled using a LabVIEW program in a computer. The force applied on the sensor was measured by attaching the sensor to a stable 1004 aluminium single point low-capacity load cell, and a voltmeter attached to the output of the load cell was used to calibrate it. The load cell gives $1.5 \mathrm{mV}$ for every $1 \mathrm{~N}$ applied and the diameter of the sensor's active area is $1 \mathrm{~cm}$. To ensure an even pressure, the contactor used to apply the pressure on the sensor was made larger than the sensor's active area.

Four different mix of Ecoflex and iron nanoparticles (1:1, $2: 1,3: 1$, and 5:1) were made to realize four different sensors. Fig. 4a shows that the Q-factor of the sensor decreases as the ratio of iron nanoparticles in Ecoflex decreases. It can also be observed that for different ratios, the inductance decreases as the ratio increases. These results are expected knowing that a higher ratio means more Ecoflex and less number of Feparticle per area.

During sensor characterization, forces from $1 \mathrm{~N}$ to $5 \mathrm{~N}$ were applied on the sensor and the change in inductance as well as motor position was recorded. The motor position helps to determine the distance the elastomer was compressed. The result shows that the distance $(\Delta \mathrm{d})$ moved by elastomer from its previous position decreased proportionally as the applied pressure increases and only $1: 1,2: 1$ and $3: 1$ saturating just around $39 \mathrm{kPa}$.

Fig. 5(b) $-5(\mathrm{e})$ show the result of the dynamic loading with a 2 second wait time and for forces between 0 to $1 \mathrm{~N}$. It was observed that the 1:1 ferromagnetic elastomer had the highest shift from the base inductance during unloading. This shift reduces as the ratio increases (Fig 5(b) - 5(e)). This means that the presence of more Ecoflex produced better results and in

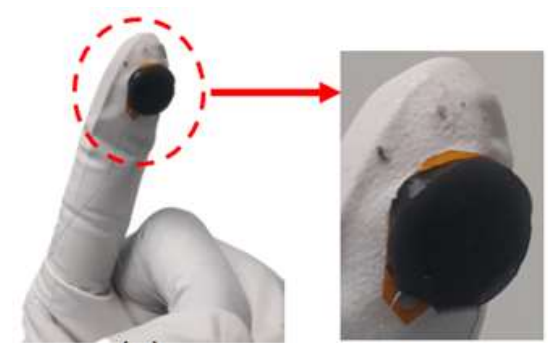

(a)

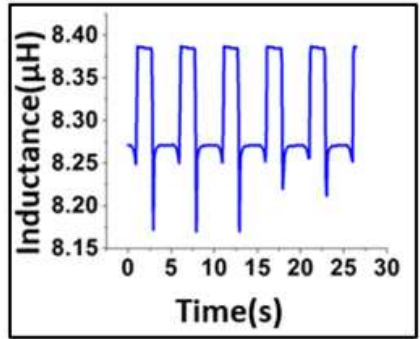

(b)

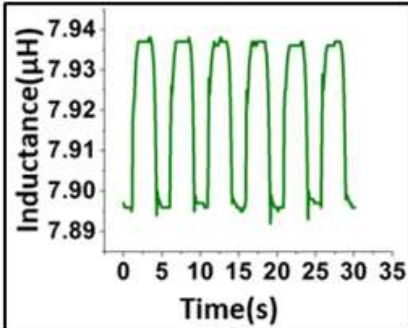

(c)

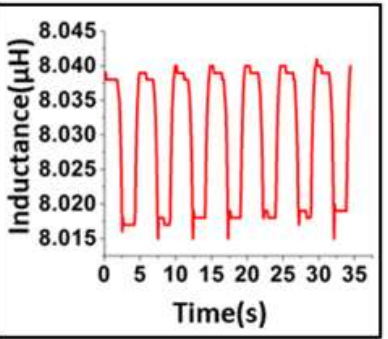

(d)

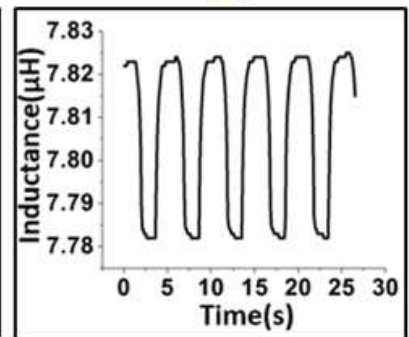

(e)
Fig. 5 Sensor and its dynamic response for different ratios of Ecoflex to Feparticles (a) Fabricated sensor on a glove (b) 1:1 (c) 2:1 (d) 3:1 (e) 5:1

this regard, the sensor with the 5:1 ferromagnetic elastomer shows the best result.

\section{CONCLUSION AND Future OUTLOOK}

A pressure sensor that works on the basis of change in inductance have been presented. The sensor shows that ratio of Ecoflex to Iron particles affects the performance of the sensor. As expected, the inductance value was highest for the sensor with the lowest ratio (1:1) of Ecoflex to iron-particles and least for that with the highest ratio (5:1). The increase in the amount of Iron particles in the polymer reduces its compressibility and hence causes the sensor to saturate faster. For deafblind application a longer range would be an advantage as it will give flexibility in coding the touch-points. Future work will be to embed the sensor in a glove with microactuator for two-way deafblind communication.

\section{ACKNOWLEDGMENT}

This work was supported in part by Engineering and Physical Sciences Research Council (EPSRC) Fellowship for Growth - Printable Tactile Skin (EP/M002527/1) project and Tertiary Education and Trust Fund (TETFund) Nigeria. 


\section{REFERENCES}

[1] R. S. Dahiya and M. Valle, Robotic Tactile Sensing: Technologies and Systems: Springer Dordrecht Heidelberg New York London, 2013.

[2] T. Yang, D. Xie, Z. Li, and H. Zhu, "Recent advances in wearable tactile sensors: Materials, sensing mechanisms, and device performance," Materials Science and Engineering: R: Reports, vol. 115, pp. 1-37, 2017.

[3] R. S. Dahiya, G. Metta, M. Valle, and G. Sandini, "Tactile sensingfrom humans to humanoids," IEEE trans. on robotics, vol. 26, pp. 1-20, 2010

[4] F. Sorgini, R. Caliò, M. C. Carrozza, and C. M. Oddo, "Haptic-assistive technologies for audition and vision sensory disabilities," Disability and Rehabilitation: Assistive Technology, pp. 1-28, 2017.

[5] M. A. Hersh, "Mobility Technologies for Blind, Partially Sighted and Deafblind People: Design Issues," in Mobility of Visually Impaired People: Fundamentals and ICT Assistive Technologies, E. Pissaloux and R. Velazquez, Eds., ed Cham: Springer International Publishing, 2018, pp. 377-409.

[6] P. Grigson, N. Lofmark, and R. Giblin, "Hand-tapper III: a prototype communication device using finger-spelling," British Journal of Visual Impairment, vol. 9, pp. 13-15, 1991.

[7] N. Caporusso, "A Wearable Malossi Alphabet Interface for Deafblind People," in AVI '08 Proceedings of the working conference on Advanced visual interfaces, Napoli Italy, 2008, pp. 445-448.

[8] U. Gollner, T. Bieling, and G. Joost, "Mobile Lorm Glove - Introducing a Communication Device for Deaf-Blind People," TEI, pp. 127 - 130, 2012.

[9] O. Ozioko, W. Taube, M. Hersh, and R. Dahiya, "SmartFingerBraille: A tactile sensing and actuation based communication glove for deafblind people," in 2017 IEEE 26th Int. Symp. on Ind. Electronics (ISIE), 2017, pp. 2014-2018.
[10] T. Choudhary, S. Kulkarni, and P. Reddy, "A Braille-based mobile communication and translation glove for deaf-blind people," in 2015 Int. Conference on Pervasive Computing (ICPC), 2015, pp. 1-4.

[11] S. Ruman and S. D. Rudrapal, "A Low-Cost Microelectromechanical Braille for blind people to communicate with blind or deaf blind people through SMS subsystem.," 3rd IEEE Int. Advanced Computing Conference(IACC), Ghaziabad, 2013.

[12] C. G. Núñez, W. T. Navaraj, E. O. Polat, and R. Dahiya, "Energy Autonomous, Flexible, and Transparent Tactile Skin," Adv. Funct. Mater., vol. 27, 2017.

[13] A. Paul, M. A. Kafi, and R. Dahiya, "Paper based pressure sensor for green electronics," in 2017 IEEE Sensors, 2017, pp. 1-3.

[14] L. Y. Chen, B. C.-K. Tee, A. L. Chortos, G. Schwartz, V. Tse, D. J. Lipomi, et al., "Continuous wireless pressure monitoring and mapping with ultra-small passive sensors for health monitoring and critical care," Nature communications, vol. 5, p. 5028, 2014.

[15] O. Ozioko, W. Taube, N. Yogeswaran, M. Hersh, and R. Dahiya "Tactile Communication System for the Interaction between Deafblind and Robots," IEEE ROMAN 2018, Nanjing, China, 2018,

[16] A. Arshad, K. A. Kadir, S. Khan, A. H. M. Z. Alam, and R. Tasnim, "A pressure sensor based inductive transducer designed for biomedical applications," in 2015 IEEE 3rd Int. Conf. on Smart Instrumentation, Measurement and Applications (ICSIMA), 2015, pp. 1-6.

[17] E. G. Bakhoum and M. H. M. Cheng, "High-Sensitivity Inductive Pressure Sensor," IEEE Trans. on Instrumentation and Measurement, vol. 60, pp. 2960-2966, 2011.

[18] R. H. Klein and R. Giblin, "A Pattern Decoding Glove for deafblind finger-spellers," British Journal of Visual Impairment, vol. 11, pp. 109$111,1993$. 